\title{
Model Sistem Pendukung Keputusan Penentuan Material Interior Furniture dengan Metode Promethee
}

\author{
Siti Sarah Abdullah \\ Universitas Suryakancana \\ Sarah0040057@gmail.com
}

\author{
Siti Sarah Nabila \\ Teknik Informatika, Universitas Suryakancana \\ sarahnabilaharyanto88@gmail.com
}

\begin{abstract}
Material Interior Furniture merupakan bahan-bahan yang digunakan dalam pembuatan interior furniture. Kualitas dari material tersebut dinilai dari beberapa aspek, salah satu aspek tersebut salah satunya ketahanan dari kayu yang digunakan, semakin mahal harga yang ditawarkan ketahanan kayu semakin tahan lama contohnya kayu jati yang dinilai mampu bertahan hingga lebih dari 20 tahun, akan tetapi kayu jati ini semakin jarang digunakan pada masa sekarang dibandingkan dengan bahan material blokmin. Sebagian besar customer tidak memahami bagaimana cara menentukan material interior furniture. Dengan budget yang disesuaikan, customer menentukan bahan furniture beserta ukuran furniture tersebut. Untuk membantu menentukan material interior furniture yang disesuaikan dengan budget dan ukuran furniture, maka dibantu dengan memodelkan Sistem Pendukung Keputusan dengan metode PROMETHEE (Prefernce Ranking Organization Method for Enrichment Evaluation). Model tersebut kemudian diuji. Hasil dari pemodelan tersebut, mempermudah pelanggan memiliki alternatif memilih material interior furniture yang sesuai dengan kebutuhan pelanggan.
\end{abstract}

Kata Kunci: Material Interior Furniture, Sistem Pendukung Keputusan, Promethee

\section{Pendahuluan}

Sistem Pendukung Keputusan merupakan sistem informasi berbasis komputer yang interaktif, fleksibel dan dapat beradaptasi, yang secara khusus dikembangkan untuk mendukung penyelesaian dari permasalahan yang tidak terstruktur untuk meningkatkan pembuatan keputusan [1] . Barang-barang furniture harganya dipengaruhi oleh bahan yang digunakan dalam pembuatannya, karena bahan yang digunakan menentukan kualitas dari barang furniture. Kualitas dari material tersebut dinilai dari beberapa aspek, salah satu aspek tersebut ketahanan dari kayu yang digunakan, semakin mahal harga yang ditawarkan ketahanan kayu semakin tahan lama contohnya kayu jati yang dinilai mampu bertahan hingga lebih dari 20 tahun, akan tetapi kayu jati ini semakin jarang digunakan pada masa sekarang dibandingkan dengan bahan material blokmin. Selain bahan, ruangan yang digunakan untuk penyimpanan furniture juga mempengaruhi harga, semakin luas ruangan semakin banyak pula bahan atau material yang digunakan, hal tersebut akan mempengaruhi harga dari furniture tersebut. Misalnya ketika pelanggan memiliki budget 4 juta rupiah sedangkan desain atau furniture yang diinginkan dengan luas ruangan yang ada bila diperhitungkan berkisar 7-8 juta rupiah, dalam hal ini produsen seringkali mendapatkan kesulitan dalam menentukan bahan yang sesuai dengan budget yang dimiliki pelanggan sekitar 4 juta tetapi dengan desain furniture yang bila diperhitungkan berkisar 7-8 juta rupiah. Berdasarkan permasalahan yang diuraikan di atas, maka dibutuhkan sistem pendukung keputusan yang dapat membantu produsen dalam menentukan material yang sesuai dengan budget pelanggan serta dapat membantu mempercepat proses tawar menawar karena tidak membutuhkan waktu lama bagi produsen dalam menentukan material yang sesuai dengan budget pelanggan. Sistem yang akan diterapkan tersebut menggunakan metode Promethee yaitu metode pengambilan keputusan penentuan urutan dalam analisis multikriteria, Promethee mempunyai kemampuan untuk menangani banyak perbandingan atau alternatif kemampuan tersebut penggunaan metode ini sesuai dengan aplikasi sistem pendukung keputusan yang akan dibuat.

\section{Landasan Teori}

a. Sistem Pendukung Keputusan

Sistem Pendukung Keputusan merupakan sistem informasi berbasis komputer yang interaktif, fleksibel dan dapat beradaptasi, yang secara khusus dikembangkan untuk mendukung penyelesaian dari permasalahan yang tidak terstruktur untuk meningkatkan pembuatan keputusan. Permasalahan yang tidak terstruktur membutuhkan kreativitas serta pertimbangan yang jauh lebih banyak. Penyelesaiannya hampir bukan merupakan pilihan antara yang benar atau salah tetapi justru yang sering terjadi ialah pilihan antara yang hampir benar dan yang mungkin salah. Pembuatan keputusan yang tidak terstruktur dibuat sebagai respon terhadap masalah-masalah yang unik, jarang dijumpai, dan tidak dapat didefinisikan secara tepat [1].

\section{b. Promethee (Preference Ranking Organizaion for Enrichment Evaluation)}

Promethee adalah suatu metode penentuan urutan (prioritas) dalam analisis multikriteria (Suryadi). Masalah pokoknya adalah kesederhanaan, kejelasan, dan kestabilan. Promethee merupakan suatu metode penentuan alternatif terbaik dalam analisis multikriteria. Hasil dari metode ini berupa perangkingan alternatif 
yang didasarkan pada kriteria-kriteria yang telah dipilih [2].

c. Material Interior Furniture

Material dikategorikan kedalam 2 (dua) jenis, yaitu [3]:

- Material alami, adalah material yang berasal dari alam, yakni: kayu, rotan, bambu

- Material buatan, yakni material yang diciptakan manusia dengan proses pengolahannya dibantu dengan teknologi mesin, contohnya: kayu lapis (polywood), fiber plastik, MDF dan HDF, logam,

\section{Metode}

Metodologi yang digunakan untuk membuat model diantaranya:

\section{a. Koleksi Data}

Data yang di koleksi diantaranya: penentuan kriteria ketahanan, kualitas bahan interior furniture, kuantitas bahan interior yang paling banyak diminati, tingkat kesulitan dalam proses pembuatan, up-to-date interior furniture

b. Pengukuran

Pengukuran dilakukan dengan kualitatif dengan mengukur data yang dikoleksi seperti pada pembahasan sebelumnya.

c. Tools untuk menganalisis

Tahapan Metode Promethee:

- Menentukan alternatif

- Menentukan kriteria

- Menentukan dominasi Kriteria dan tipe preferensi

- Menghitung nilai indeks preferensi

- Perankingan

d. Model Sistem Pendukung Keputusan dengan Promethee

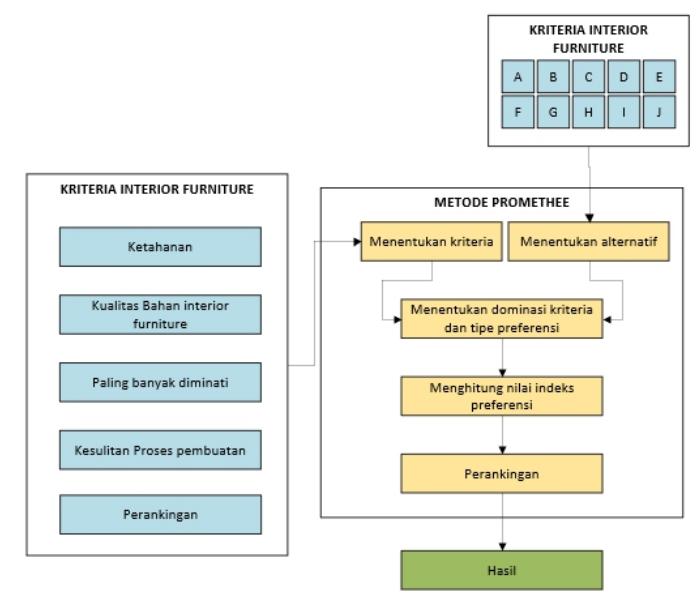

Gambar 1 model sistem pendukung keputusan dengan metode promethee

\section{Hasil dan Pembahasan}

a. Menentukan alternatif (material )

Berikut alternatif material

$\mathrm{A}=$ Kayu Jati, Engsel/Rel Laci otomatis, HPL ++ (Rp.3.000.000)

$\mathrm{B}=$ Kayu Jati, Engsel/Rel Laci Otomatis, HPL + (Rp.2.900.000)
$\mathrm{C}=$ Kayu Jati, Engsel/Rel Laci standar, HPL + (Rp. 2.700.000)

$\mathrm{D}=$ Kayu Jati, Engsel/Rel Laci Standar, HPL (Rp.2.600.000)

$\mathrm{E}=$ Multi + , Engsel/Rel Laci otomatis, HPL $+($ Rp. 2.000.000)

$\mathrm{F}=$ Multi + , Engsel/Rel Laci standar, HPL + (Rp. 1.900.000)

$\mathrm{G}=$ Multi + , Engsel/Rel Laci standar, HPL (Rp. 1.800.000)

$\mathrm{H}=$ Blockmin, Multi Standar, Engsel/Rel Laci otomatis, HPL + (Rp.1.700.000)

$\mathrm{I}=$ Blockmin, Multi standar, Engsel/Rel Laci standar, $\mathrm{HPL}+$ (Rp. 1.600.000)

$\mathrm{J}=$ Blockmin, Multi standar, Engsel/Rel Laci standar, HPL (Rp. 1.500.000)

b. Menentukkan Kriteria

\begin{tabular}{|c|c|c|c|}
\hline $\mathrm{K}$ & Kriteria & Tingkat & Nilai \\
\hline \multirow[t]{3}{*}{ K1 } & \multirow[t]{3}{*}{ Ketahanan } & $\begin{array}{l}\text { Sangat } \\
\text { tahan } \\
\text { lama }\end{array}$ & 3 \\
\hline & & $\begin{array}{l}\text { Tahan } \\
\text { Lama }\end{array}$ & 2 \\
\hline & & $\begin{array}{l}\text { Kurang } \\
\text { tahan } \\
\text { lama }\end{array}$ & 1 \\
\hline \multirow[t]{3}{*}{$\mathrm{K} 2$} & \multirow{3}{*}{$\begin{array}{l}\text { Kualitas } \\
\text { High } \\
\text { Pressure } \\
\text { Laminate }\end{array}$} & $\begin{array}{l}\text { Sangat } \\
\text { Bagus }\end{array}$ & 3 \\
\hline & & Bagus & 2 \\
\hline & & $\begin{array}{l}\text { Cukup } \\
\text { Bagus }\end{array}$ & 1 \\
\hline \multirow[t]{3}{*}{ K3 } & \multirow{3}{*}{$\begin{array}{l}\text { Paling } \\
\text { banyak } \\
\text { diminati }\end{array}$} & $\begin{array}{l}\text { Sangat } \\
\text { diminati }\end{array}$ & 3 \\
\hline & & Diminati & 2 \\
\hline & & $\begin{array}{l}\text { Tidak } \\
\text { diminati }\end{array}$ & 1 \\
\hline \multirow[t]{3}{*}{ K4 } & \multirow{3}{*}{$\begin{array}{l}\text { Proses } \\
\text { Pembuatan }\end{array}$} & Mudah & 3 \\
\hline & & Sedang & 2 \\
\hline & & Sulit & 1 \\
\hline \multirow[t]{3}{*}{ K5 } & \multirow[t]{3}{*}{ Trendi } & Terbaru & 3 \\
\hline & & Baru & 2 \\
\hline & & lama & 1 \\
\hline
\end{tabular}

c. Menentukan dominasi kriteria dan tipe preferensi

\begin{tabular}{|l|l|l|l|l|l|l|l|l|l|l|}
\hline \multirow{2}{*}{ Kriteria } & \multicolumn{10}{|c|}{} \\
\cline { 2 - 12 } & A & B & C & D & E & F & G & H & I & J \\
\hline K1 & 3 & 3 & 3 & 3 & 2 & 2 & 2 & 1 & 1 & 1 \\
\hline K2 & 3 & 2 & 2 & 1 & 2 & 2 & 1 & 2 & 2 & 1 \\
\hline K3 & 1 & 2 & 1 & 1 & 2 & 2 & 2 & 2 & 3 & 3 \\
\hline K4 & 1 & 1 & 1 & 1 & 2 & 3 & 3 & 3 & 3 & 3 \\
\hline K5 & 1 & 1 & 1 & 1 & 2 & 2 & 2 & 3 & 3 & 3 \\
\hline
\end{tabular}

Keterangan : Menggunakan Tipe Preferensi pertama yaitu Usual (Biasa)

d. Menghitung Nilai Indeks Preferensi

Preferensi Kriteria Usual

Nilai Preferensi A

$(\mathrm{A}, \mathrm{B})=\mathrm{K} 1=3-3=0 \mathrm{H}(\mathrm{d})=0$ $\mathrm{K} 2=3-2=1 \mathrm{H}(\mathrm{d})=1$ 


\begin{tabular}{|c|c|}
\hline $\mathrm{K} 3=1-2=-1 \mathrm{H}(\mathrm{d})=0$ & $\mathrm{~K} 4=1-1=0 \mathrm{H}(\mathrm{d})=0$ \\
\hline $\mathrm{K} 4=1-1=0 \mathrm{H}(\mathrm{d})=0$ & $\mathrm{~K} 5=1-1=0 \mathrm{H}(\mathrm{d})=0$ \\
\hline $\mathrm{K} 5=1-1=0 \mathrm{H}(\mathrm{d})=0$ & $\mathrm{IP}=1 / 5^{*}(0+0+1+0+0)=0.2$ \\
\hline $\mathrm{IP}=1 / 5^{*}(0+1+0+0+0)=0.2$ & $(\mathrm{~B}, \mathrm{D})=\mathrm{K} 1=3-3=0 \mathrm{H}(\mathrm{d})=0$ \\
\hline$(\mathrm{A}, \mathrm{C})=\mathrm{K} 1=3-3=0 \mathrm{H}(\mathrm{d})=0$ & $\mathrm{~K} 2=2-1=1 \mathrm{H}(\mathrm{d})=1$ \\
\hline $\mathrm{K} 2=3-2=1 \mathrm{H}(\mathrm{d})=1$ & $\mathrm{~K} 3=2-1=1 \mathrm{H}(\mathrm{d})=1$ \\
\hline $\mathrm{K} 3=1-1=0 \mathrm{H}(\mathrm{d})=0$ & $\mathrm{~K} 4=1-1=0 \mathrm{H}(\mathrm{d})=0$ \\
\hline $\mathrm{K} 4=1-1=0 \mathrm{H}(\mathrm{d})=0$ & $\mathrm{~K} 5=1-1=0 \mathrm{H}(\mathrm{d})=0$ \\
\hline $\mathrm{K} 5=1-1=0 \mathrm{H}(\mathrm{d})=0$ & $\mathrm{IP}=1 / 5 *(0+1+1+0+0)=0.4$ \\
\hline $\mathrm{IP}=1 / 5 *(0+1+0+0+0)=0.2$ & $(\mathrm{~B}, \mathrm{E})=\mathrm{K} 1=3-2=1 \mathrm{H}(\mathrm{d})=1$ \\
\hline$(A, D)=K 1=3-3=0 H(d)=0$ & $\mathrm{~K} 2=2-2=0 \mathrm{H}(\mathrm{d})=0$ \\
\hline $\mathrm{K} 2=3-1=2 \mathrm{H}(\mathrm{d})=1$ & $\mathrm{~K} 3=2-2=0 \mathrm{H}(\mathrm{d})=0$ \\
\hline $\mathrm{K} 3=1-1=0 \mathrm{H}(\mathrm{d})=0$ & $\mathrm{~K} 4=1-2=-1 \mathrm{H}(\mathrm{d})=0$ \\
\hline $\mathrm{K} 4=1-1=0 \mathrm{H}(\mathrm{d})=0$ & $\mathrm{~K} 5=1-2=-1 \mathrm{H}(\mathrm{d})=0$ \\
\hline $\mathrm{K} 5=1-1=0 \mathrm{H}(\mathrm{d})=0$ & $\mathrm{IP}=1 / 5^{*}(1+0+0+0+0)=0.2$ \\
\hline $\mathrm{IP}=1 / 5^{*}(0+1+0+0+0)=0.2$ & $(\mathrm{~B}, \mathrm{~F})=\mathrm{K} 1=3-2=1 \mathrm{H}(\mathrm{d})=1$ \\
\hline$(\mathrm{A}, \mathrm{E})=\mathrm{K} 1=3-2=1 \mathrm{H}(\mathrm{d})=1$ & $\mathrm{~K} 2=2-2=0 \mathrm{H}(\mathrm{d})=0$ \\
\hline $\mathrm{K} 2=3-2=1 \mathrm{H}(\mathrm{d})=1$ & $\mathrm{~K} 3=2-2=0 \mathrm{H}(\mathrm{d})=1$ \\
\hline $\mathrm{K} 3=1-2=-1 \mathrm{H}(\mathrm{d})=0$ & $\mathrm{~K} 4=1-3=-2 \mathrm{H}(\mathrm{d})=0$ \\
\hline $\mathrm{K} 4=1-2=-1 \mathrm{H}(\mathrm{d})=0$ & $K 5=1-2=-1 H(d)=0$ \\
\hline $\mathrm{K} 5=1-2=-1 \mathrm{H}(\mathrm{d})=0$ & $\mathrm{IP}=1 / 5 *(1+0+0+0+0)=0.2$ \\
\hline $\mathrm{IP}=1 / 5^{*}(1+1+0+0+0)=0.4$ & $(\mathrm{~B}, \mathrm{G})=\mathrm{K} 1=3-2=1 \mathrm{H}(\mathrm{d})=1$ \\
\hline$(\mathrm{A}, \mathrm{F})=\mathrm{K} 1=3-2=1 \mathrm{H}(\mathrm{d})=1$ & $\mathrm{~K} 2=2-1=1 \mathrm{H}(\mathrm{d})=1$ \\
\hline $\mathrm{K} 2=3-2=1 \mathrm{H}(\mathrm{d})=1$ & $\mathrm{~K} 3=2-2=0 \mathrm{H}(\mathrm{d})=0$ \\
\hline $\mathrm{K} 3=1-2=-1 \mathrm{H}(\mathrm{d})=0$ & $\mathrm{~K} 4=1-3=-2 \mathrm{H}(\mathrm{d})=0$ \\
\hline $\mathrm{K} 4=1-3=-2 \mathrm{H}(\mathrm{d})=0$ & $\mathrm{~K} 5=1-2=-1 \mathrm{H}(\mathrm{d})=0$ \\
\hline $\mathrm{K} 5=1-2=-1 \mathrm{H}(\mathrm{d})=0$ & $\mathrm{IP}=1 / 5^{*}(1+1+0+0+0)=0.4$ \\
\hline $\mathrm{IP}=1 / 5 *(1+1+0+0+0)=0.4$ & $(\mathrm{~B}, \mathrm{H})=\mathrm{K} 1=3-1=2 \mathrm{H}(\mathrm{d})=1$ \\
\hline$(\mathrm{A}, \mathrm{G})=\mathrm{K} 1=3-2=1 \mathrm{H}(\mathrm{d})=1$ & $\mathrm{~K} 2=2-2=0 \mathrm{H}(\mathrm{d})=0$ \\
\hline $\mathrm{K} 2=3-1=2 \mathrm{H}(\mathrm{d})=1$ & $\mathrm{~K} 3=2-2=0 \mathrm{H}(\mathrm{d})=0$ \\
\hline $\mathrm{K} 3=1-2=-1 \mathrm{H}(\mathrm{d})=0$ & $\mathrm{~K} 4=1-3=-2 \mathrm{H}(\mathrm{d})=0$ \\
\hline $\mathrm{K} 4=1-3=-2 \mathrm{H}(\mathrm{d})=0$ & $\mathrm{~K} 5=1-3=-2 \mathrm{H}(\mathrm{d})=0$ \\
\hline $\mathrm{K} 5=1-2=-1 \mathrm{H}(\mathrm{d})=0$ & $\mathrm{IP}=1 / 5^{*}(1+0+0+0+0)=0.2$ \\
\hline $\mathrm{IP}=1 / 5^{*}(1+1+0+0+0)=0.4$ & $(\mathrm{~B}, \mathrm{I})=\mathrm{K} 1=3-1=2 \mathrm{H}(\mathrm{d})=1$ \\
\hline$(\mathrm{A}, \mathrm{H})=\mathrm{K} 1=3-1=2 \mathrm{H}(\mathrm{d})=1$ & $\mathrm{~K} 2=2-2=0 \mathrm{H}(\mathrm{d})=0$ \\
\hline $\mathrm{K} 2=3-2=1 \mathrm{H}(\mathrm{d})=1$ & $\mathrm{~K} 3=2-3=-1 \mathrm{H}(\mathrm{d})=0$ \\
\hline $\mathrm{K} 3=1-2=-1 \mathrm{H}(\mathrm{d})=0$ & $\mathrm{~K} 4=1-3=-2 \mathrm{H}(\mathrm{d})=0$ \\
\hline $\mathrm{K} 4=1-3=-2 \mathrm{H}(\mathrm{d})=0$ & $\mathrm{~K} 5=1-3=-2 \mathrm{H}(\mathrm{d})=0$ \\
\hline $\mathrm{K} 5=1-3=-2 \mathrm{H}(\mathrm{d})=0$ & $\mathrm{IP}=1 / 5 *(1+0+0+0+0)=0.2$ \\
\hline $\mathrm{IP}=1 / 5 *(1+1+0+0+0)=0.4$ & $(\mathrm{~B}, \mathrm{~J})=\mathrm{K} 1=3-1=2 \mathrm{H}(\mathrm{d})=1$ \\
\hline$(\mathrm{A}, \mathrm{I})=\mathrm{K} 1=3-1=1 \mathrm{H}(\mathrm{d})=1$ & $\mathrm{~K} 2=2-1=1 \mathrm{H}(\mathrm{d})=1$ \\
\hline $\mathrm{K} 2=3-2=1 \mathrm{H}(\mathrm{d})=1$ & $\mathrm{~K} 3=2-3=-1 \mathrm{H}(\mathrm{d})=0$ \\
\hline $\mathrm{K} 3=1-3=-2 \mathrm{H}(\mathrm{d})=0$ & $\mathrm{~K} 4=1-3=-2 \mathrm{H}(\mathrm{d})=0$ \\
\hline $\mathrm{K} 4=1-3=-2 \mathrm{H}(\mathrm{d})=0$ & $\mathrm{~K} 5=1-3=-2 \mathrm{H}(\mathrm{d})=0$ \\
\hline $\mathrm{K} 5=1-3=-2 \mathrm{H}(\mathrm{d})=0$ & $\mathrm{IP}=1 / 5^{*}(1+1+0+0+0)=0.4$ \\
\hline \multicolumn{2}{|l|}{$\mathrm{IP}=1 / 5 *(1+1+0+0+0)=0.4$} \\
\hline$(\mathrm{A}, \mathrm{J})=\mathrm{K} 1=3-1=2 \mathrm{H}(\mathrm{d})=1$ & Nilai Preferensi C \\
\hline $\mathrm{K} 2=3-1=2 \mathrm{H}(\mathrm{d})=1$ & $(\mathrm{C}, \mathrm{A})=\mathrm{K} 1=3-3=0 \mathrm{H}(\mathrm{d})=0$ \\
\hline $\mathrm{K} 3=1-3=-2 \mathrm{H}(\mathrm{d})=0$ & $\mathrm{~K} 2=2-3=-1 \mathrm{H}(\mathrm{d})=0$ \\
\hline $\mathrm{K} 4=1-3=-2 \mathrm{H}(\mathrm{d})=0$ & $\mathrm{~K} 3=1-1=0 \mathrm{H}(\mathrm{d})=0$ \\
\hline$K 5=1-3=-2 H(d)=0$ & $\mathrm{~K} 4=1-1=0 \mathrm{H}(\mathrm{d})=0$ \\
\hline \multirow{2}{*}{$\mathrm{IP}=1 / 5^{*}(1+1+0+0+0)=0.4$} & $\mathrm{~K} 5=1-1=0 \mathrm{H}(\mathrm{d})=0$ \\
\hline & $\mathrm{IP}=1 / 5 *(0+0+0+0+0)=0$ \\
\hline Nilai Preferensi B & $(\mathrm{C}, \mathrm{B})=\mathrm{K} 1=3-3=0 \mathrm{H}(\mathrm{d})=0$ \\
\hline$(\mathrm{B}, \mathrm{A})=\mathrm{K} 1=3-3=0 \mathrm{H}(\mathrm{d})=0$ & $\mathrm{~K} 2=2-2=0 \mathrm{H}(\mathrm{d})=0$ \\
\hline $\mathrm{K} 2=2-3=-1 \mathrm{H}(\mathrm{d})=0$ & $\mathrm{~K} 3=1-2=-1 \mathrm{H}(\mathrm{d})=0$ \\
\hline $\mathrm{K} 3=2-1=1 \mathrm{H}(\mathrm{d})=1$ & $\mathrm{~K} 4=1-1=0 \mathrm{H}(\mathrm{d})=0$ \\
\hline $\mathrm{K} 4=1-1=0 \mathrm{H}(\mathrm{d})=0$ & $\mathrm{~K} 5=1-1=0 \mathrm{H}(\mathrm{d})=0$ \\
\hline $\mathrm{K} 5=1-1=0 \mathrm{H}(\mathrm{d})=0$ & $\mathrm{IP}=1 / 5 *(0+0+0+0+0)=0$ \\
\hline $\mathrm{IP}=1 / 5 *(0+0+1+0+0)=0.2$ & $(C, D)=K 1=3-3=0 H(d)=0$ \\
\hline$(\mathrm{B}, \mathrm{C})=\mathrm{K} 1=3-3=0 \mathrm{H}(\mathrm{d})=0$ & $\mathrm{~K} 2=2-1=1 \mathrm{H}(\mathrm{d})=1$ \\
\hline $\mathrm{K} 2=2-2=0 \mathrm{H}(\mathrm{d})=0$ & $\mathrm{~K} 3=1-1=0 \mathrm{H}(\mathrm{d})=0$ \\
\hline $\mathrm{K} 3=2-1=1 \mathrm{H}(\mathrm{d})=1$ & $\mathrm{~K} 4=1-1=0 \mathrm{H}(\mathrm{d})=0$ \\
\hline
\end{tabular}




\begin{tabular}{|c|c|}
\hline $\mathrm{K} 5=1-1=0 \mathrm{H}(\mathrm{d})=0$ & $\mathrm{IP}=1 / 5 *(1+0+0+0+0)=0.2$ \\
\hline $\mathrm{IP}=1 / 5^{*}(0+1+0+0+0)=0.2$ & $(\mathrm{D}, \mathrm{F})=\mathrm{K} 1=3-2=1 \mathrm{H}(\mathrm{d})=1$ \\
\hline$(\mathrm{C}, \mathrm{E})=\mathrm{K} 1=3-2=1 \mathrm{H}(\mathrm{d})=1$ & $\mathrm{~K} 2=1-2=-1 \mathrm{H}(\mathrm{d})=0$ \\
\hline $\mathrm{K} 2=2-2=0 \mathrm{H}(\mathrm{d})=0$ & $\mathrm{~K} 3=1-2=-1 \mathrm{H}(\mathrm{d})=0$ \\
\hline $\mathrm{K} 3=1-2=-1 \mathrm{H}(\mathrm{d})=0$ & $\mathrm{~K} 4=1-3=-2 \mathrm{H}(\mathrm{d})=0$ \\
\hline $\mathrm{K} 4=1-2=-1 \mathrm{H}(\mathrm{d})=0$ & $K 5=1-2=-1 H(d)=0$ \\
\hline $\mathrm{K} 5=1-2=-1 \mathrm{H}(\mathrm{d})=0$ & $\mathrm{IP}=1 / 5^{*}(1+0+0+0+0)=0.2$ \\
\hline $\mathrm{IP}=1 / 5 *(1+0+0+0+0)=0.2$ & $(\mathrm{D}, \mathrm{G})=\mathrm{K} 1=3-2=1 \mathrm{H}(\mathrm{d})=1$ \\
\hline$(\mathrm{C}, \mathrm{F})=\mathrm{K} 1=3-2=1 \mathrm{H}(\mathrm{d})=1$ & $\mathrm{~K} 2=1-1=0 \mathrm{H}(\mathrm{d})=0$ \\
\hline $\mathrm{K} 2=2-2=0 \mathrm{H}(\mathrm{d})=0$ & $\mathrm{~K} 3=1-2=-1 \mathrm{H}(\mathrm{d})=0$ \\
\hline $\mathrm{K} 3=1-2=-1 \mathrm{H}(\mathrm{d})=0$ & $\mathrm{~K} 4=1-3=-2 \mathrm{H}(\mathrm{d})=0$ \\
\hline $\mathrm{K} 4=1-3=-2 \mathrm{H}(\mathrm{d})=0$ & $\mathrm{~K} 5=1-2=-1 \mathrm{H}(\mathrm{d})=0$ \\
\hline $\mathrm{K} 5=1-2=-1 \mathrm{H}(\mathrm{d})=0$ & $\mathrm{IP}=1 / 5^{*}(1+0+0+0+0)=0.2$ \\
\hline $\mathrm{IP}=1 / 5 *(1+0+0+0+0)=0.2$ & $(\mathrm{D}, \mathrm{H})=\mathrm{K} 1=3-1=2 \mathrm{H}(\mathrm{d})=1$ \\
\hline$(\mathrm{C}, \mathrm{G})=\mathrm{K} 1=3-2=1 \mathrm{H}(\mathrm{d})=1$ & $\mathrm{~K} 2=1-2=-1 \mathrm{H}(\mathrm{d})=0$ \\
\hline $\mathrm{K} 2=2-1=1 \mathrm{H}(\mathrm{d})=1$ & $\mathrm{~K} 3=1-2=-1 \mathrm{H}(\mathrm{d})=0$ \\
\hline $\mathrm{K} 3=1-2=-1 \mathrm{H}(\mathrm{d})=0$ & $\mathrm{~K} 4=1-3=-2 \mathrm{H}(\mathrm{d})=0$ \\
\hline $\mathrm{K} 4=1-3=-2 \mathrm{H}(\mathrm{d})=0$ & $\mathrm{~K} 5=1-3=-2 \mathrm{H}(\mathrm{d})=0$ \\
\hline $\mathrm{K} 5=1-2=-1 \mathrm{H}(\mathrm{d})=0$ & $\mathrm{IP}=1 / 5 *(1+0+0+0+0)=0.2$ \\
\hline $\mathrm{IP}=1 / 5^{*}(1+1+0+0+0)=0.4$ & $(\mathrm{D}, \mathrm{I})=\mathrm{K} 1=3-1=2 \mathrm{H}(\mathrm{d})=1$ \\
\hline$(\mathrm{C}, \mathrm{H})=\mathrm{K} 1=3-1=2 \mathrm{H}(\mathrm{d})=1$ & $\mathrm{~K} 2=1-2=-1 \mathrm{H}(\mathrm{d})=0$ \\
\hline $\mathrm{K} 2=2-2=0 \mathrm{H}(\mathrm{d})=0$ & $\mathrm{~K} 3=1-3=-2 \mathrm{H}(\mathrm{d})=0$ \\
\hline $\mathrm{K} 3=1-2=-1 \mathrm{H}(\mathrm{d})=0$ & $\mathrm{~K} 4=1-3=-2 \mathrm{H}(\mathrm{d})=0$ \\
\hline $\mathrm{K} 4=1-3=-2 \mathrm{H}(\mathrm{d})=0$ & $\mathrm{~K} 5=1-3=-2 \mathrm{H}(\mathrm{d})=0$ \\
\hline $\mathrm{K} 5=1-3=-2 \mathrm{H}(\mathrm{d})=0$ & $\mathrm{IP}=1 / 5 *(1+0+0+0+0)=0.2$ \\
\hline $\mathrm{IP}=1 / 5 *(1+0+0+0+0)=0.2$ & $(\mathrm{D}, \mathrm{J})=\mathrm{K} 1=3-1=2 \mathrm{H}(\mathrm{d})=1$ \\
\hline$(\mathrm{C}, \mathrm{I})=\mathrm{K} 1=3-1=2 \mathrm{H}(\mathrm{d})=1$ & $\mathrm{~K} 2=1-1=0 \mathrm{H}(\mathrm{d})=0$ \\
\hline $\mathrm{K} 2=2-2=0 \mathrm{H}(\mathrm{d})=0$ & $\mathrm{~K} 3=1-3=-2 \mathrm{H}(\mathrm{d})=0$ \\
\hline $\mathrm{K} 3=1-3=-2 \mathrm{H}(\mathrm{d})=0$ & $\mathrm{~K} 4=1-3=-1 \mathrm{H}(\mathrm{d})=0$ \\
\hline $\mathrm{K} 4=1-3=-2 \mathrm{H}(\mathrm{d})=0$ & $\mathrm{~K} 5=1-3=-1 \mathrm{H}(\mathrm{d})=0$ \\
\hline $\mathrm{K} 5=1-3=-2 \mathrm{H}(\mathrm{d})=0$ & $\mathrm{IP}=1 / 5^{*}(1+0+0+0+0)=0.2$ \\
\hline $\mathrm{IP}=1 / 5 *(1+0+0+0+0)=0.2$ & Nilai Preferensi E \\
\hline$(\mathrm{C}, \mathrm{J})=\mathrm{K} 1=3-1=2 \mathrm{H}(\mathrm{d})=1$ & $(\mathrm{E}, \mathrm{A})=\mathrm{K} 1=2-3=-1 \mathrm{H}(\mathrm{d})=0$ \\
\hline $\mathrm{K} 2=2-1=1 \mathrm{H}(\mathrm{d})=1$ & $\mathrm{~K} 2=2-3=-1 \mathrm{H}(\mathrm{d})=0$ \\
\hline $\mathrm{K} 3=1-3=-2 \mathrm{H}(\mathrm{d})=0$ & $\mathrm{~K} 3=2-1=1 \mathrm{H}(\mathrm{d})=1$ \\
\hline $\mathrm{K} 4=1-3=-2 \mathrm{H}(\mathrm{d})=0$ & $\mathrm{~K} 4=2-1=1 \mathrm{H}(\mathrm{d})=1$ \\
\hline $\mathrm{K} 5=1-3=-2 \mathrm{H}(\mathrm{d})=0$ & $\mathrm{~K} 5=2-1=1 \mathrm{H}(\mathrm{d})=1$ \\
\hline $\mathrm{IP}=1 / 5 *(1+1+0+0+0)=0.4$ & $\mathrm{IP}=1 / 5 *(0+0+1+1+1)-=0.6$ \\
\hline & $(\mathrm{E}, \mathrm{B})=\mathrm{K} 1=2-3=-1 \mathrm{H}(\mathrm{d})=0$ \\
\hline Nilai Preferensi D & $\mathrm{K} 2=2-2=0 \mathrm{H}(\mathrm{d})=0$ \\
\hline$(\mathrm{D}, \mathrm{A})=\mathrm{K} 1=3-3=0 \mathrm{H}(\mathrm{d})=0$ & $\mathrm{~K} 3=2-2=0 \mathrm{H}(\mathrm{d})=0$ \\
\hline $\mathrm{K} 2=1-3=-2 \mathrm{H}(\mathrm{d})=0$ & $\mathrm{~K} 4=2-1=1 \mathrm{H}(\mathrm{d})=1$ \\
\hline $\mathrm{K} 3=1-1=0 \mathrm{H}(\mathrm{d})=0$ & $\mathrm{~K} 5=2-1=1 \mathrm{H}(\mathrm{d})=1$ \\
\hline $\mathrm{K} 4=1-1=0 \mathrm{H}(\mathrm{d})=0$ & $\mathrm{IP}=1 / 5 *(0+0+0+1+1)-=0.4$ \\
\hline $\mathrm{K} 5=1-1=0 \mathrm{H}(\mathrm{d})=0$ & $(\mathrm{E}, \mathrm{C})=\mathrm{K} 1=2-3=-1 \mathrm{H}(\mathrm{d})=0$ \\
\hline $\mathrm{IP}=1 / 5 *(0+0+0+0+0)=0$ & $\mathrm{~K} 2=2-2=0 \mathrm{H}(\mathrm{d})=0$ \\
\hline$(\mathrm{D}, \mathrm{B})=\mathrm{K} 1=3-3=0 \mathrm{H}(\mathrm{d})=0$ & $\mathrm{~K} 3=2-1=1 \mathrm{H}(\mathrm{d})=1$ \\
\hline $\mathrm{K} 2=1-2=-1 \mathrm{H}(\mathrm{d})=0$ & $\mathrm{~K} 4=2-1=1 \mathrm{H}(\mathrm{d})=1$ \\
\hline $\mathrm{K} 3=1-2=-1 \mathrm{H}(\mathrm{d})=0$ & $\mathrm{~K} 5=2-1=1 \mathrm{H}(\mathrm{d})=1$ \\
\hline $\mathrm{K} 4=1-1=0 \mathrm{H}(\mathrm{d})=0$ & $\mathrm{IP}=1 / 5^{*}(0+0+1+1+1)=0.6$ \\
\hline $\mathrm{K} 5=1-1=0 \mathrm{H}(\mathrm{d})=0$ & $(E, D)=K 1=2-3=-1 H(d)=0$ \\
\hline $\mathrm{IP}=1 / 5 *(0+0+0+0+0)=0$ & $\mathrm{~K} 2=2-1=1 \mathrm{H}(\mathrm{d})=1$ \\
\hline$(D, C)=K 1=3-3=0 H(d)=0$ & $\mathrm{~K} 3=2-1=1 \mathrm{H}(\mathrm{d})=1$ \\
\hline $\mathrm{K} 2=1-2=-1 \mathrm{H}(\mathrm{d})=0$ & $\mathrm{~K} 4=2-1=1 \mathrm{H}(\mathrm{d})=1$ \\
\hline $\mathrm{K} 3=1-1=0 \mathrm{H}(\mathrm{d})=0$ & $\mathrm{~K} 5=2-1=1 \mathrm{H}(\mathrm{d})=1$ \\
\hline $\mathrm{K} 4=1-1=0 \mathrm{H}(\mathrm{d})=0$ & $\mathrm{IP}=1 / 5^{*}(0+1+1+1+1)=0.8$ \\
\hline $\mathrm{K} 5=1-1=0 \mathrm{H}(\mathrm{d})=0$ & $(\mathrm{E}, \mathrm{F})=\mathrm{K} 1=2-2=0 \mathrm{H}(\mathrm{d})=0$ \\
\hline $\mathrm{IP}=1 / 5 *(0+0+0+0+0)=0$ & $\mathrm{~K} 2=2-2=0 \mathrm{H}(\mathrm{d})=0$ \\
\hline$(\mathrm{D}, \mathrm{E})=\mathrm{K} 1=3-2=1 \mathrm{H}(\mathrm{d})=1$ & $\mathrm{~K} 3=2-2=0 \mathrm{H}(\mathrm{d})=0$ \\
\hline $\mathrm{K} 2=1-2=-1 \mathrm{H}(\mathrm{d})=0$ & $\mathrm{~K} 4=2-3=-1 \mathrm{H}(\mathrm{d})=0$ \\
\hline $\mathrm{K} 3=1-2=-1 \mathrm{H}(\mathrm{d})=0$ & $\mathrm{~K} 5=2-2=0 \mathrm{H}(\mathrm{d})=0$ \\
\hline $\mathrm{K} 4=1-2=-1 \mathrm{H}(\mathrm{d})=0$ & $\mathrm{IP}=1 / 5 *(0+0+0+0+0)=0$ \\
\hline $\mathrm{K} 5=1-2=-1 \mathrm{H}(\mathrm{d})=0$ & $(\mathrm{E}, \mathrm{G})=\mathrm{K} 1=2-2=0 \mathrm{H}(\mathrm{d})=0$ \\
\hline
\end{tabular}




\begin{tabular}{|c|c|}
\hline $\mathrm{K} 2=2-1=1 \mathrm{H}(\mathrm{d})=1$ & $\mathrm{~K} 2=2-2=0 \mathrm{H}(\mathrm{d})=0$ \\
\hline $\mathrm{K} 3=2-2=0 \mathrm{H}(\mathrm{d})=0$ & $\mathrm{~K} 3=2-2=0 \mathrm{H}(\mathrm{d})=0$ \\
\hline $\mathrm{K} 4=2-3=-1 \mathrm{H}(\mathrm{d})=0$ & $\mathrm{~K} 4=3-3=0 \mathrm{H}(\mathrm{d})=0$ \\
\hline $\mathrm{K} 5=2-3=-1 \mathrm{H}(\mathrm{d})=0$ & $\mathrm{~K} 5=2-3=-1 \mathrm{H}(\mathrm{d})=0$ \\
\hline $\mathrm{IP}=1 / 5 *(0+1+0+0+0)=0.2$ & $\mathrm{IP}=1 / 5 *(1+0+0+0+0)=0.2$ \\
\hline$(\mathrm{E}, \mathrm{H})=\mathrm{K} 1=2-1=1 \mathrm{H}(\mathrm{d})=1$ & $(\mathrm{~F}, \mathrm{I})=\mathrm{K} 1=2-1=1 \mathrm{H}(\mathrm{d})=1$ \\
\hline $\mathrm{K} 2=2-2=0 \mathrm{H}(\mathrm{d})=0$ & $\mathrm{~K} 2=2-2=0 \mathrm{H}(\mathrm{d})=0$ \\
\hline $\mathrm{K} 3=2-2=0 \mathrm{H}(\mathrm{d})=0$ & $\mathrm{~K} 3=2-3=-1 \mathrm{H}(\mathrm{d})=0$ \\
\hline $\mathrm{K} 4=2-3=-1 \mathrm{H}(\mathrm{d})=0$ & $\mathrm{~K} 4=3-3=0 \mathrm{H}(\mathrm{d})=0$ \\
\hline $\mathrm{K} 5=2-3=-1 \mathrm{H}(\mathrm{d})=0$ & $\mathrm{~K} 5=2-3=-1 \mathrm{H}(\mathrm{d})=0$ \\
\hline $\mathrm{IP}=1 / 5 *(1+0+0+0+0)=0.2$ & $\mathrm{IP}=1 / 5 *(1+0+0+0+0)=0.2$ \\
\hline$(\mathrm{E}, \mathrm{I})=\mathrm{K} 1=2-1=1 \mathrm{H}(\mathrm{d})=1$ & $(\mathrm{~F}, \mathrm{~J})=\mathrm{K} 1=2-1=1 \mathrm{H}(\mathrm{d})=1$ \\
\hline $\mathrm{K} 2=2-2=0 \mathrm{H}(\mathrm{d})=0$ & $\mathrm{~K} 2=2-1=1 \mathrm{H}(\mathrm{d})=1$ \\
\hline $\mathrm{K} 3=2-3=-1 \mathrm{H}(\mathrm{d})=0$ & $\mathrm{~K} 3=2-3=-1 \mathrm{H}(\mathrm{d})=0$ \\
\hline $\mathrm{K} 4=2-3=-1 \mathrm{H}(\mathrm{d})=0$ & $\mathrm{~K} 4=3-3=0 \mathrm{H}(\mathrm{d})=0$ \\
\hline $\mathrm{K} 5=2-3=-1 \mathrm{H}(\mathrm{d})=0$ & $\mathrm{~K} 5=2-3=-1 \mathrm{H}(\mathrm{d})=0$ \\
\hline $\mathrm{IP}=1 / 5 *(1+0+0+0+0)=0.2$ & $\mathrm{IP}=1 / 5 *(1+1+0+0+0)=0.4$ \\
\hline \multicolumn{2}{|l|}{$(\mathrm{E}, \mathrm{J})=\mathrm{K} 1=2-1=-1 \mathrm{H}(\mathrm{d})=0$} \\
\hline $\mathrm{K} 2=2-1=0 \mathrm{H}(\mathrm{d})=1$ & Nilai Preferensi G \\
\hline $\mathrm{K} 3=2-3=-1 \mathrm{H}(\mathrm{d})=0$ & $(\mathrm{G}, \mathrm{A})=\mathrm{K} 1=2-3=-1 \mathrm{H}(\mathrm{d})=0$ \\
\hline $\mathrm{K} 4=2-3=-1 \mathrm{H}(\mathrm{d})=0$ & $\mathrm{~K} 2=1-3=-2 \mathrm{H}(\mathrm{d})=0$ \\
\hline $\mathrm{K} 5=2-3=-1 \mathrm{H}(\mathrm{d})=0$ & $\mathrm{~K} 3=2-1=1 \mathrm{H}(\mathrm{d})=1$ \\
\hline \multirow[t]{3}{*}{$\mathrm{IP}=1 / 5 *(0+1+0+0+0)=0.2$} & $\mathrm{~K} 4=3-1=2 \mathrm{H}(\mathrm{d})=1$ \\
\hline & $\mathrm{K} 5=2-1=1 \mathrm{H}(\mathrm{d})=1$ \\
\hline & $\mathrm{IP}=1 / 5^{*}(0+0+1+1+1)=0.6$ \\
\hline Nilai Preferensi F & $(\mathrm{G}, \mathrm{B})=\mathrm{K} 1=2-3=-1 \mathrm{H}(\mathrm{d})=0$ \\
\hline$(\mathrm{F}, \mathrm{A})=\mathrm{K} 1=2-3=-1 \mathrm{H}(\mathrm{d})=0$ & $\mathrm{~K} 2=1-2=-1 \mathrm{H}(\mathrm{d})=0$ \\
\hline $\mathrm{K} 2=2-3=-1 \mathrm{H}(\mathrm{d})=0$ & $\mathrm{~K} 3=2-2=0 \mathrm{H}(\mathrm{d})=0$ \\
\hline $\mathrm{K} 3=2-1=1 \mathrm{H}(\mathrm{d})=1$ & $\mathrm{~K} 4=3-1=2 \mathrm{H}(\mathrm{d})=1$ \\
\hline$K 4=3-1=2 H(d)=1$ & $\mathrm{~K} 5=2-1=1 \mathrm{H}(\mathrm{d})=1$ \\
\hline $\mathrm{K} 5=2-1=1 \mathrm{H}(\mathrm{d})=1$ & $\mathrm{IP}=1 / 5^{*}(0+0+0+1+1)=0.4$ \\
\hline $\mathrm{IP}=1 / 5 *(0+0+1+1+1)=0.6$ & $(\mathrm{G}, \mathrm{C})=\mathrm{K} 1=2-3=-1 \mathrm{H}(\mathrm{d})=0$ \\
\hline$(\mathrm{F}, \mathrm{B})=\mathrm{K} 1=2-3=-1 \mathrm{H}(\mathrm{d})=0$ & $\mathrm{~K} 2=1-2=-1 \mathrm{H}(\mathrm{d})=0$ \\
\hline $\mathrm{K} 2=2-2=0 \mathrm{H}(\mathrm{d})=0$ & $\mathrm{~K} 3=2-1=1 \mathrm{H}(\mathrm{d})=1$ \\
\hline $\mathrm{K} 3=2-2=0 \mathrm{H}(\mathrm{d})=0$ & $\mathrm{~K} 4=3-1=2 \mathrm{H}(\mathrm{d})=1$ \\
\hline$K 4=3-1=2 H(d)=1$ & $\mathrm{~K} 5=2-1=1 \mathrm{H}(\mathrm{d})=1$ \\
\hline $\mathrm{K} 5=2-1=1 \mathrm{H}(\mathrm{d})=1$ & $\mathrm{IP}=1 / 5 *(0+0+1+1+1)=0.6$ \\
\hline $\mathrm{IP}=1 / 5^{*}(0+0+0+1+1)=0.4$ & $(\mathrm{G}, \mathrm{D})=\mathrm{K} 1=2-3=-1 \mathrm{H}(\mathrm{d})=0$ \\
\hline$(\mathrm{F}, \mathrm{C})=\mathrm{K} 1=2-3=-1 \mathrm{H}(\mathrm{d})=0$ & $\mathrm{~K} 2=1-1=0 \mathrm{H}(\mathrm{d})=0$ \\
\hline $\mathrm{K} 2=2-2=0 \mathrm{H}(\mathrm{d})=0$ & $\mathrm{~K} 3=2-1=1 \mathrm{H}(\mathrm{d})=1$ \\
\hline $\mathrm{K} 3=2-1=1 \mathrm{H}(\mathrm{d})=1$ & $\mathrm{~K} 4=3-1=2 \mathrm{H}(\mathrm{d})=1$ \\
\hline $\mathrm{K} 4=3-1=2 \mathrm{H}(\mathrm{d})=1$ & $\mathrm{~K} 5=2-1=1 \mathrm{H}(\mathrm{d})=1$ \\
\hline $\mathrm{K} 5=2-1=1 \mathrm{H}(\mathrm{d})=1$ & $\mathrm{IP}=1 / 5 *(0+0+1+1+1)=0.6$ \\
\hline $\mathrm{IP}=1 / 5^{*}(0+0+1+1+1)=0.6$ & $(\mathrm{G}, \mathrm{E})=\mathrm{K} 1=2-2=0 \mathrm{H}(\mathrm{d})=0$ \\
\hline$(F, D)=K 1=2-3=-1 H(d)=0$ & $\mathrm{~K} 2=1-2=-1 \mathrm{H}(\mathrm{d})=0$ \\
\hline $\mathrm{K} 2=2-1=1 \mathrm{H}(\mathrm{d})=1$ & $\mathrm{~K} 3=2-2=0 \mathrm{H}(\mathrm{d})=0$ \\
\hline $\mathrm{K} 3=2-1=1 \mathrm{H}(\mathrm{d})=1$ & $\mathrm{~K} 4=3-2=1 \mathrm{H}(\mathrm{d})=1$ \\
\hline $\mathrm{K} 4=3-1=2 \mathrm{H}(\mathrm{d})=1$ & $\mathrm{~K} 5=2-2=0 \mathrm{H}(\mathrm{d})=0$ \\
\hline $\mathrm{K} 5=2-1=1 \mathrm{H}(\mathrm{d})=1$ & $\mathrm{IP}=1 / 5 *(0+0+0+1+0)=0.4$ \\
\hline $\mathrm{IP}=1 / 5^{*}(0+1+1+1+1)=0.8$ & $(\mathrm{G}, \mathrm{F})=\mathrm{K} 1=2-2=0 \mathrm{H}(\mathrm{d})=0$ \\
\hline$(\mathrm{F}, \mathrm{E})=\mathrm{K} 1=2-2=0 \mathrm{H}(\mathrm{d})=0$ & $\mathrm{~K} 2=1-2=-1 \mathrm{H}(\mathrm{d})=0$ \\
\hline $\mathrm{K} 2=2-2=0 \mathrm{H}(\mathrm{d})=0$ & $\mathrm{~K} 3=2-2=0 \mathrm{H}(\mathrm{d})=0$ \\
\hline $\mathrm{K} 3=2-2=0 \mathrm{H}(\mathrm{d})=0$ & $\mathrm{~K} 4=3-3=0 \mathrm{H}(\mathrm{d})=0$ \\
\hline $\mathrm{K} 4=3-2=1 \mathrm{H}(\mathrm{d})=1$ & $\mathrm{~K} 5=2-2=0 \mathrm{H}(\mathrm{d})=0$ \\
\hline $\mathrm{K} 5=2-2=0 \mathrm{H}(\mathrm{d})=0$ & $\mathrm{IP}=1 / 5 *(0+0+0+0+0)=0$ \\
\hline $\mathrm{IP}=1 / 5^{*}(0+0+0+1+0)=0.2$ & $(\mathrm{G}, \mathrm{H})=\mathrm{K} 1=2-1=1 \mathrm{H}(\mathrm{d})=1$ \\
\hline$(\mathrm{F}, \mathrm{G})=\mathrm{K} 1=2-2=0 \mathrm{H}(\mathrm{d})=0$ & $\mathrm{~K} 2=1-2=-1 \mathrm{H}(\mathrm{d})=0$ \\
\hline $\mathrm{K} 2=2-1=1 \mathrm{H}(\mathrm{d})=1$ & $\mathrm{~K} 3=2-2=0 \mathrm{H}(\mathrm{d})=0$ \\
\hline $\mathrm{K} 3=2-2=0 \mathrm{H}(\mathrm{d})=0$ & $\mathrm{~K} 4=3-3=0 \mathrm{H}(\mathrm{d})=0$ \\
\hline $\mathrm{K} 4=3-3=0 \mathrm{H}(\mathrm{d})=0$ & $\mathrm{~K} 5=2-3=-1 \mathrm{H}(\mathrm{d})=0$ \\
\hline $\mathrm{K} 5=2-2=0 \mathrm{H}(\mathrm{d})=0$ & $\mathrm{IP}=1 / 5^{*}(1+0+0+0+0)=0.2$ \\
\hline $\mathrm{IP}=1 / 5 *(0+1+0+0+0)=0.2$ & $(\mathrm{G}, \mathrm{I})=\mathrm{K} 1=2-1=1 \mathrm{H}(\mathrm{d})=1$ \\
\hline$(\mathrm{F}, \mathrm{H})=\mathrm{K} 1=2-1=1 \mathrm{H}(\mathrm{d})=1$ & $\mathrm{~K} 2=1-2=-1 \mathrm{H}(\mathrm{d})=0$ \\
\hline
\end{tabular}




\begin{tabular}{|c|c|}
\hline $\mathrm{K} 3=2-3=-1 \mathrm{H}(\mathrm{d})=0$ & $\mathrm{~K} 4=3-3=0 \mathrm{H}(\mathrm{d})=0$ \\
\hline $\mathrm{K} 4=3-3=0 \mathrm{H}(\mathrm{d})=0$ & $\mathrm{~K} 5=3-3=0 \mathrm{H}(\mathrm{d})=0$ \\
\hline $\mathrm{K} 5=2-3=-1 \mathrm{H}(\mathrm{d})=0$ & $\mathrm{IP}=1 / 5 *(0+1+0+0+0)=0.2$ \\
\hline \multicolumn{2}{|l|}{$\mathrm{IP}=1 / 5^{*}(1+0+0+0+0)=0.2$} \\
\hline$(\mathrm{G}, \mathrm{J})=\mathrm{K} 1=2-1=1 \mathrm{H}(\mathrm{d})=1$ & Nilai Preferensi I \\
\hline $\mathrm{K} 2=1-1=0 \mathrm{H}(\mathrm{d})=0$ & $(\mathrm{I}, \mathrm{A})=\mathrm{K} 1=1-3=-2 \mathrm{H}(\mathrm{d})=0$ \\
\hline $\mathrm{K} 3=2-3=-1 \mathrm{H}(\mathrm{d})=0$ & $\mathrm{~K} 2=2-3=-1 \mathrm{H}(\mathrm{d})=0$ \\
\hline $\mathrm{K} 4=3-3=0 \mathrm{H}(\mathrm{d})=0$ & $\mathrm{~K} 3=3-1=2 \mathrm{H}(\mathrm{d})=1$ \\
\hline $\mathrm{K} 5=2-3=-1 \mathrm{H}(\mathrm{d})=0$ & $\mathrm{~K} 4=3-1=2 \mathrm{H}(\mathrm{d})=1$ \\
\hline \multirow[t]{2}{*}{$\mathrm{IP}=1 / 5^{*}(1+0+0+0+0)=0.2$} & $\mathrm{~K} 5=3-1=2 \mathrm{H}(\mathrm{d})=1$ \\
\hline & $\mathrm{IP}=1 / 5 *(0+0+1+1+1)=0.6$ \\
\hline Nilai Preferensi H & $(\mathrm{I}, \mathrm{B})=\mathrm{K} 1=1-3=-2 \mathrm{H}(\mathrm{d})=0$ \\
\hline$(\mathrm{H}, \mathrm{A})=\mathrm{K} 1=1-3=-2 \mathrm{H}(\mathrm{d})=0$ & $\mathrm{~K} 2=2-2=0 \mathrm{H}(\mathrm{d})=0$ \\
\hline $\mathrm{K} 2=2-3=-1 \mathrm{H}(\mathrm{d})=0$ & $\mathrm{~K} 3=3-2=1 \mathrm{H}(\mathrm{d})=1$ \\
\hline $\mathrm{K} 3=2-1=1 \mathrm{H}(\mathrm{d})=1$ & $\mathrm{~K} 4=3-1=1 \mathrm{H}(\mathrm{d})=1$ \\
\hline $\mathrm{K} 4=3-1=2 \mathrm{H}(\mathrm{d})=1$ & $\mathrm{~K} 5=3-1=1 \mathrm{H}(\mathrm{d})=1$ \\
\hline $\mathrm{K} 5=3-1=2 \mathrm{H}(\mathrm{d})=1$ & $\mathrm{IP}=1 / 5^{*}(0+0+1+1+1)=0.6$ \\
\hline $\mathrm{IP}=1 / 5^{*}(0+0+1+1+1)=0.6$ & $(\mathrm{I}, \mathrm{C})=\mathrm{K} 1=1-3=-2 \mathrm{H}(\mathrm{d})=0$ \\
\hline$(\mathrm{H}, \mathrm{B})=\mathrm{K} 1=1-3=-2 \mathrm{H}(\mathrm{d})=0$ & $\mathrm{~K} 2=2-2=0 \mathrm{H}(\mathrm{d})=0$ \\
\hline $\mathrm{K} 2=2-2=0 \mathrm{H}(\mathrm{d})=0$ & $\mathrm{~K} 3=3-1=2 \mathrm{H}(\mathrm{d})=1$ \\
\hline $\mathrm{K} 3=2-2=0 \mathrm{H}(\mathrm{d})=0$ & $\mathrm{~K} 4=3-1=2 \mathrm{H}(\mathrm{d})=1$ \\
\hline $\mathrm{K} 4=3-1=2 \mathrm{H}(\mathrm{d})=1$ & $\mathrm{~K} 5=3-1=2 \mathrm{H}(\mathrm{d})=1$ \\
\hline $\mathrm{K} 5=3-1=2 \mathrm{H}(\mathrm{d})=1$ & $\mathrm{IP}=1 / 5^{*}(0+0+1+1+1)=0.6$ \\
\hline $\mathrm{IP}=1 / 5 *(0+0+0+1+1)=0.4$ & $(\mathrm{I}, \mathrm{D})=\mathrm{K} 1=1-3=-2 \mathrm{H}(\mathrm{d})=0$ \\
\hline$(\mathrm{H}, \mathrm{C})=\mathrm{K} 1=1-3=-2 \mathrm{H}(\mathrm{d})=0$ & $\mathrm{~K} 2=2-1=1 \mathrm{H}(\mathrm{d})=1$ \\
\hline $\mathrm{K} 2=2-2=0 \mathrm{H}(\mathrm{d})=0$ & $\mathrm{~K} 3=3-1=2 \mathrm{H}(\mathrm{d})=1$ \\
\hline $\mathrm{K} 3=2-1=1 \mathrm{H}(\mathrm{d})=1$ & $\mathrm{~K} 4=3-1=2 \mathrm{H}(\mathrm{d})=1$ \\
\hline $\mathrm{K} 4=3-1=2 \mathrm{H}(\mathrm{d})=1$ & $\mathrm{~K} 5=3-1=2 \mathrm{H}(\mathrm{d})=1$ \\
\hline $\mathrm{K} 5=3-1=2 \mathrm{H}(\mathrm{d})=1$ & $\mathrm{IP}=1 / 5 *(0+1+1+1+1)=0.8$ \\
\hline $\mathrm{IP}=1 / 5 *(0+0+1+1+1)=0.6$ & $(\mathrm{I}, \mathrm{E})=\mathrm{K} 1=1-2=-1 \mathrm{H}(\mathrm{d})=0$ \\
\hline$(H, D)=K 1=1-3=-2 H(d)=0$ & $\mathrm{~K} 2=2-2=0 \mathrm{H}(\mathrm{d})=0$ \\
\hline $\mathrm{K} 2=2-1=1 \mathrm{H}(\mathrm{d})=1$ & $\mathrm{~K} 3=3-2=1 \mathrm{H}(\mathrm{d})=1$ \\
\hline $\mathrm{K} 3=2-1=1 \mathrm{H}(\mathrm{d})=1$ & $\mathrm{~K} 4=3-2=1 \mathrm{H}(\mathrm{d})=1$ \\
\hline $\mathrm{K} 4=3-1=2 \mathrm{H}(\mathrm{d})=1$ & $\mathrm{~K} 5=3-2=1 \mathrm{H}(\mathrm{d})=1$ \\
\hline $\mathrm{K} 5=3-1=2 \mathrm{H}(\mathrm{d})=1$ & $\mathrm{IP}=1 / 5 *(0+0+1+1+1)=0.6$ \\
\hline $\mathrm{IP}=1 / 5^{*}(0+1+1+1+1)=0.8$ & $(\mathrm{I}, \mathrm{F})=\mathrm{K} 1=1-2=-1 \mathrm{H}(\mathrm{d})=0$ \\
\hline$(\mathrm{H}, \mathrm{E})=\mathrm{K} 1=1-2=-1 \mathrm{H}(\mathrm{d})=0$ & $\mathrm{~K} 2=2-2=0 \mathrm{H}(\mathrm{d})=0$ \\
\hline $\mathrm{K} 2=2-2=0 \mathrm{H}(\mathrm{d})=0$ & $\mathrm{~K} 3=3-2=1 \mathrm{H}(\mathrm{d})=1$ \\
\hline $\mathrm{K} 3=2-2=0 \mathrm{H}(\mathrm{d})=0$ & $\mathrm{~K} 4=3-3=0 \mathrm{H}(\mathrm{d})=0$ \\
\hline $\mathrm{K} 4=3-2=1 \mathrm{H}(\mathrm{d})=1$ & $\mathrm{~K} 5=3-2=1 \mathrm{H}(\mathrm{d})=1$ \\
\hline $\mathrm{K} 5=3-2=1 \mathrm{H}(\mathrm{d})=1$ & $\mathrm{IP}=1 / 5 *(0+0+1+0+1)=0.4$ \\
\hline $\mathrm{IP}=1 / 5^{*}(0+0+0+1+1)=0.4$ & $(\mathrm{I}, \mathrm{G})=\mathrm{K} 1=1-2=-1 \mathrm{H}(\mathrm{d})=0$ \\
\hline$(H, F)=K 1=1-2=-1 H(d)=0$ & $\mathrm{~K} 2=2-1=-1 \mathrm{H}(\mathrm{d})=0$ \\
\hline $\mathrm{K} 2=2-2=0 \mathrm{H}(\mathrm{d})=0$ & $\mathrm{~K} 3=3-2=1 \mathrm{H}(\mathrm{d})=1$ \\
\hline $\mathrm{K} 3=2-2=0 \mathrm{H}(\mathrm{d})=0$ & $\mathrm{~K} 4=3-3=0 \mathrm{H}(\mathrm{d})=0$ \\
\hline $\mathrm{K} 4=3-3=0 \mathrm{H}(\mathrm{d})=0$ & $\mathrm{~K} 5=3-2=1 \mathrm{H}(\mathrm{d})=1$ \\
\hline $\mathrm{K} 5=3-2=1 \mathrm{H}(\mathrm{d})=1$ & $\mathrm{IP}=1 / 5^{*}(0+0+1+0+1)=0.4$ \\
\hline $\mathrm{IP}=1 / 5 *(0+0+0+0+1)=0.2$ & $(\mathrm{I}, \mathrm{H})=\mathrm{K} 1=1-1=0 \mathrm{H}(\mathrm{d})=0$ \\
\hline$(\mathrm{H}, \mathrm{G})=\mathrm{K} 1=1-2=-1 \mathrm{H}(\mathrm{d})=0$ & $\mathrm{~K} 2=2-2=0 \mathrm{H}(\mathrm{d})=0$ \\
\hline $\mathrm{K} 2=2-1=1 \mathrm{H}(\mathrm{d})=1$ & $\mathrm{~K} 3=3-2=1 \mathrm{H}(\mathrm{d})=1$ \\
\hline $\mathrm{K} 3=2-2=0 \mathrm{H}(\mathrm{d})=0$ & $\mathrm{~K} 4=3-3=0 \mathrm{H}(\mathrm{d})=0$ \\
\hline $\mathrm{K} 4=3-3=0 \mathrm{H}(\mathrm{d})=0$ & $\mathrm{~K} 5=3-3=1 \mathrm{H}(\mathrm{d})=1$ \\
\hline $\mathrm{K} 5=3-2=1 \mathrm{H}(\mathrm{d})=1$ & $\mathrm{IP}=1 / 5^{*}(0+0+1+0+1)=0.4$ \\
\hline $\mathrm{IP}=1 / 5^{*}(0+1+0+0+1)=0.4$ & $(\mathrm{~J}, \mathrm{~A})=\mathrm{K} 1=1-1=0 \mathrm{H}(\mathrm{d})=0$ \\
\hline$(\mathrm{H}, \mathrm{I})=\mathrm{K} 1=1-1=0 \mathrm{H}(\mathrm{d})=0$ & $\mathrm{~K} 2=2-1=1 \mathrm{H}(\mathrm{d})=1$ \\
\hline $\mathrm{K} 2=2-2=0 \mathrm{H}(\mathrm{d})=0$ & $\mathrm{~K} 3=3-3=0 \mathrm{H}(\mathrm{d})=0$ \\
\hline $\mathrm{K} 3=2-3=-1 \mathrm{H}(\mathrm{d})=0$ & $\mathrm{~K} 4=3-3=0 \mathrm{H}(\mathrm{d})=0$ \\
\hline $\mathrm{K} 4=3-3=0 \mathrm{H}(\mathrm{d})=0$ & $\mathrm{~K} 5=3-3=0 \mathrm{H}(\mathrm{d})=0$ \\
\hline $\mathrm{K} 5=3-3=0 \mathrm{H}(\mathrm{d})=0$ & $\mathrm{IP}=1 / 5 *(0+1+0+0+0)=0.2$ \\
\hline \multicolumn{2}{|l|}{$\mathrm{IP}=1 / 5 *(0+0+0+0+0)=0$} \\
\hline$(\mathrm{H}, \mathrm{J})=\mathrm{K} 1=1-1=0 \mathrm{H}(\mathrm{d})=0$ & Nilai Preferensi J \\
\hline $\mathrm{K} 2=2-1=1 \mathrm{H}(\mathrm{d})=1$ & $(\mathrm{~J}, \mathrm{~A})=\mathrm{K} 1=1-3=-2 \mathrm{H}(\mathrm{d})=0$ \\
\hline $\mathrm{K} 3=2-3=-1 \mathrm{H}(\mathrm{d})=0$ & $\mathrm{~K} 2=1-3=-2 \mathrm{H}(\mathrm{d})=0$ \\
\hline
\end{tabular}




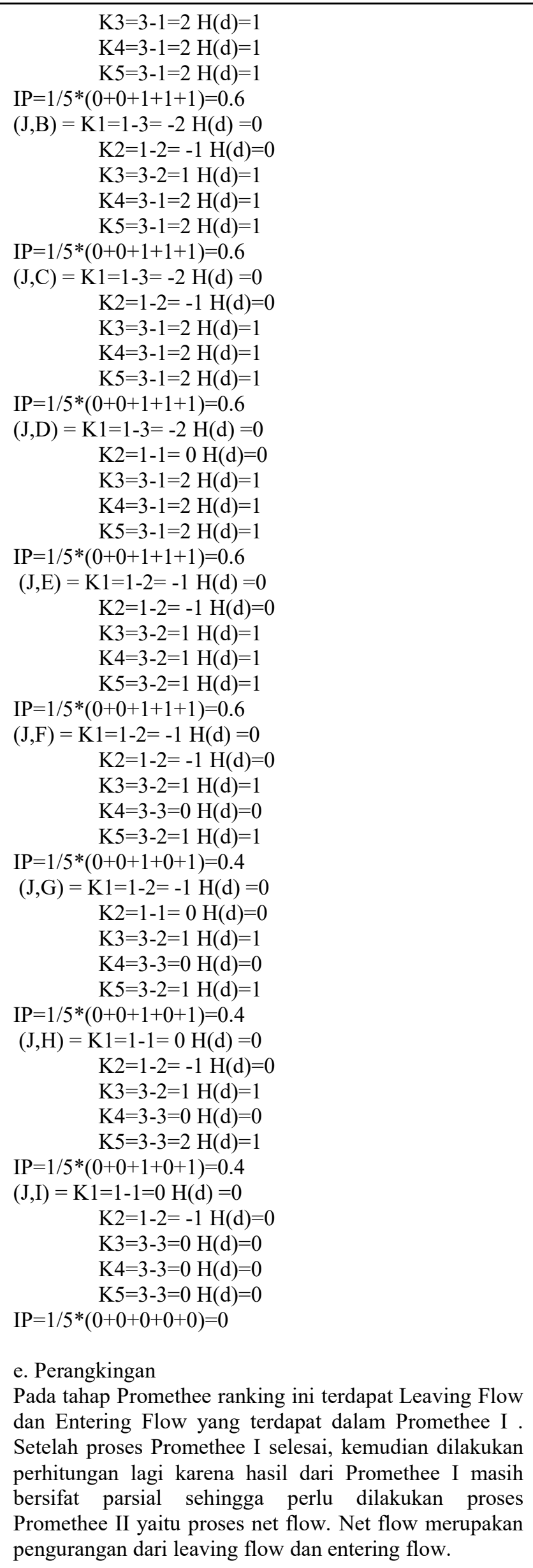

Perhitungan Promethee I :

a. Leaving Flow:

$\mathrm{A}=1 /(10-1) *(0.2+0.2+0.2+0.4+0.4+0.4+0.4+$ $0.4+0.4)=0.333$

$\mathrm{B}=1 /(10-1) *(0.2+0.2+0.4+0.2+0.2+0.4+0.2+$ $0.2+0.4)=0.267$

$\mathrm{C}=1 /(10-1) *(0+0+0.2+0.2+0.2+0.4+0.4+0.2+$ $0.4)=0.222$

$\mathrm{D}=1 /(10-1) *(0+0+0+0.2+0.2+0.2+0.2+0.2+$

$0.4)=0.156$

$\mathrm{E}=1 /(10-1) *(0.6+0.4+0.6+0.8+0+0.2+0.2+0.2$ $+0.4)=0.378$

$\mathrm{F}=1 /(10-1) *(0.6+0.4+0.6+0.8+0.2+0.2+0.2+$

$0.2+0.4)=0.4$

$\mathrm{G}=1 /(10-1) *(0.6+0.4+0.6+0.6+0.2+0+0.2+0.2$ $+0.2)=0.333$

$\mathrm{H}=1 /(10-1) *(0.6+0.4+0.6+0.8+0.4+0.2+0.4+0$

$+0.2)=0.4$

$\mathrm{I}=1 /(10-1) *(0.6+0.6+0.6+0.8+0.6+0.4+0.6+$

$0.2+0.2)=0.511$

$\mathrm{J}=1 /(10-1) *(0.6+0.6+0.6+0.6+0.6+0.2+0.4+0.2$

$+0)=0.422$

b. Entering Flow:

$\mathrm{A}=1 /(10-1) *(0.2+0+0+0.6+0.6+0.6+0.6+0.6+$ $0.6)=0.422$

$\mathrm{B}=1 /(10-1) *(0.2+0+0+0.4+0.4+0.4+0.4+0.6+$ $0.6)=0.333$

$\mathrm{C}=1 /(10-1) *(0.2+0.2+0+0.6+0.6+0.6+0.6+0.6$

$+0.6)=0.444$

$\mathrm{D}=1 /(10-1) *(0.2+0.4+0.2+0.8+0.8+0.6+0.8+$

$0.8+0.6)=0.578$

$\mathrm{E}=1 /(10-1) *(0.4+0.2+0.2+0.2+0+0.2+0.4+0.6$

$+0.6)=0.333$

$\mathrm{F}=1 /(10-1) *(0.4+0.2+0.2+0.2+0+0+0.2+0.4+$ $0.2)=0.2$

$\mathrm{G}=1 /(10-1) *(0.4+0.4+0.4+0.2+0.2+0.2+0.4+$

$0.6+0.4)=0.356$

$\mathrm{H}=1 /(10-1) *(0.4+0.2+0.4+0.2+0.2+0.2+0.2+$ $0.2+0.2)=0.244$

$\mathrm{I}=1 /(10-1) *(0.4+0.2+0.2+0.2+0.2+0.2+0.2+0$

$+0)=0.178$

$\mathrm{J}=1 /(10-1) *(0.4+0.4+0.4+0.4+0.4+0.4+0.2+0.2$ $+0.2)=0.333$

c. Net Flow

$\mathrm{A}=0.333-0.422=-0.089$

$\mathrm{B}=0.267-0.333=-0.067$

$\mathrm{C}=0.222-0.444=-0.222$

$\mathrm{D}=0.156-0.578=-0.422$

$\mathrm{E}=0.378-0.333=0.044$

$\mathrm{F}=0.4-0.2 \quad=0.2$

$\mathrm{G}=0.333-0.356=-0.022$

$\mathrm{H}=0.4-0.244=0.156$

$\mathrm{I}=0.511-0.178=0.333$

$\mathrm{J}=0.422-0.333=0.089$

Jadi Alternatif yang tertinggi yaitu Alternatif I dengan hasil 0.333 


\section{Kesimpulan}

Penelitian yang dihasilkan dengan membuat model menggunakan metodologi Sistem Pendukung Keputusan dengan Promethee telah membuktikan hasil perhitungan yang sesuai dengan kriteria pelanggan, yaitu dengan mempertimbangkan kriteria sesuai dengan budget yang dimiliki oleh pelanggan.

\section{Daftar Pustaka}

[1] A. S. Honggowibowo and T. Sediartie, "Sistem Pendukung Keputusan Pemilihan Desain Interior Menggunakan Metode Analytical Hierarchy Process," TELKOMNIKA, pp. 49-56, 2004.

[2] R. Anjasmaya and S. Andayani, "Sistem Pendukung Keputusan Penentuan Komoditi Sayuran Berdasarkan Karakteristik Lahan Menggunakan Metode PROMETHEE," JUITA, vol. VI, no. 2, 2018.

[3] b. Arif and D. Susilawati, "Penggunaan Material Furniture sebagai Pendukung Tema pada Desain Interior Nanny's Pavillon di Bandung," Jurnal Rekajiva, vol. 1, no. 2, 2013. 